\title{
GENERIC PROPERTIES OF EIGENFUNCTIONS OF ELLIPTIC PARTIAL DIFFERENTIAL OPERATORS
}

BY

\author{
JEFFREY H. ALBERT
}

\begin{abstract}
The problem considered here is that of describing generically the zeros, critical points and critical values of eigenfunctions of elliptic partial differential operators. We consider operators of the form $L+\rho$, where $L$ is a fixed, second-order, selfadjoint, $C^{\infty}$ linear elliptic partial differential operator on a compact manifold (without boundary) and $\rho$ is a $C^{\infty}$ function. It is shown that, for almost all $\rho$, i.e. for a residual set, the eigenvalues of $L+\rho$ are simple and the eigenfunctions have the following properties: (1) they are Morse functions; (2) distinct critical points have distinct critical values; (3) 0 is not a critical value.
\end{abstract}

1. Introduction. For eigenfunctions of ordinary differential equations of Sturm-Liouville type, the zero and critical point sets are extremely simple to describe and have very nice properties. Each consists of isolated points, critical points do not lie in the zero set and the second derivative never vanishes at a critical point. These are trivial consequences of the uniqueness theorem. In higher dimensions the situation is quite different. Asking that the zeros be isolated is unreasonable, of course. But it is plausible to hope for the other properties ("second derivative" must be replaced by "Hessian") for a function satisfying an appropriate partial differential equation. Still, there are simple examples in which these fail, e.g. for the Laplacian on the 2-sphere [2]. However, for eigenfunctions of "most" operators, they do hold.

More precisely, let $M$ be a $C^{\infty}$ manifold of dimension $\nu$, compact, connected and without boundary. $C^{\infty}(M)$ denotes the real-valued $C^{\infty}$ functions on $M$. For $u \in C^{\infty}(M)$, the zero or nodal set of $u$ is $u^{-1}(0)=\{x \in M: u(x)=$ $0\} . a \in M$ is a critical point of $u$ if $d u(a)=0$, where $d u=$ $\left(\partial u / \partial x_{1}, \ldots, \partial u / \partial x_{v}\right)$ in local coordinates. $b \in \mathbf{R}$ is a critical value of $u$ if there exists a critical point $a \in M$ with $u(a)=b$. A critical point $a \in M$ is nondegenerate if the Hessian matrix $\left(\left(\partial^{2} u(a) / \partial x_{i} \partial x_{j}\right)\right)$ is nonsingular. We are interested in the following properties of $u \in C^{\infty}(M)$ :

(E1) $u$ is a Morse function, i.e. all critical points are nondegenerate;

(E2) distinct critical points have distinct critical values;

(E3) 0 is not a critical value, i.e. there are no nodal critical points.

Received by the editors November 4, 1976.

AMS (MOS) subject classifications (1970). Primary 35J15, 35B05; Secondary 35P99, 35B20, 57D70, 58G99. 
Let $L$ be a fixed, second-order, selfadjoint, $C^{\infty}$ linear elliptic partial differential operator on $M$.

MAIN THEOREM. $\left\{\rho \in C^{\infty}(M)\right.$ : all nonzero eigenfunctions of $L+\rho$ satisfy (E1), (E2), (E3)\} contains a residual set in $C^{\infty}(M)$. (Residual means a countable intersection of open, dense sets.)

This theorem was first announced by the author some time ago [2] for $\operatorname{dim} M=2$. The proof, contained in the author's thesis [3], used Sobolev space estimates and perturbation theory. The restriction to $\operatorname{dim} M=2$ was due to a lemma proved using complex variables (see Lemma 3.4). Later, $\mathrm{K}$. Uhlenbeck showed, using infinite dimensional transversality techniques, that the theorem does hold in all dimensions [9], [10].

The aim of the current paper is to present the author's original proof for $\operatorname{dim} M=2(\$ \S 2,3,4,7)$, a Sard's theorem argument based on Uhlenbeck's ideas to extend this proof to higher dimensions (\$5) and some comments on the two methods (\$6). One advantage of this type of analytic argument is that it enables one to find the generic operators, eigenvalues and eigenfunctions explicitly. Finally, the use of a manifold without boundary and the strong smoothness requirements serve mainly to simplify the presentation; they can easily be relaxed.

For the proof, the main theorem is broken down as follows: The eigenvalues of $L+\rho$ can be written in an increasing sequence $\lambda_{1} \leqslant \lambda_{2}$ $\leqslant \ldots \leqslant \lambda_{n} \leqslant \ldots \rightarrow+\infty$ in which each eigenvalue is repeated according to its multiplicity. ( $L$ is assumed to be normalized so its spectrum is bounded from below, and by eigenvalue we mean $\lambda$ satisfies $\operatorname{ker}(L+\rho-\lambda) \neq 0$; e.g. $L=-\Delta$.)

Let $A_{n}=\left\{\rho \in C^{\infty}(M)\right.$ : the first $n$ eigenvalues of $L+\rho$ are simple $\}$. Then $A_{n}$ is open and dense in $C^{\infty}(M)$ for all $n$ [1]. Let $B_{n}=\left\{\rho \in A_{n}\right.$ : nonzero eigenfunctions corresponding to the first $n$ eigenvalues satisfy E1, E2 and E3\}, with $B_{0}=C^{\infty}(M)$. The requirement that the eigenvalues be simple is made to allow one to deal with each eigenspace by considering only one nonzero eigenfunction, for properties (E1), (E2) and (E3) are unchanged under multiplication by a constant.

THEOREM 1.1. $B_{n}$ is open in $C^{\infty}(M)$ for all $n$.

Theorem 1.2. $B_{n}$ is dense in $A_{n} \cap B_{n-1}$ for $n=1,2, \ldots$

Using Theorem 1.2 and the density of $A_{n}$, it follows by induction that $B_{n}$ is dense in $C^{\infty}(M)$. Hence $\bigcap_{n=1}^{\infty} B_{n}=\left\{\rho \in C^{\infty}(M)\right.$ : all eigenvalues of $L+\rho$ are simple and all nonzero eigenfunctions satisfy E1, E2, E3 $\}$ is residual, which implies the main theorem.

Theorem 1.1 is proved in $\S 2$. Theorem 1.2 is set up in such a way that we 
need to show only that we can perturb the operator so that the $n$th eigenfunction satisfies E1, E2 and E3. The proof has two parts. First we discuss how to perturb an operator to make the $n$th eigenfunction satisfy E3. This is the hard part. $\$ 3$ contains information on the growth of eigenfunctions near nodal critical points if $\operatorname{dim} M=2$. $\$ 4$ uses this information to give the original proof in dimension 2 that nodal critical points can be perturbed away. In $\$ 5$, a Sard's theorem argument is used to extend this method to higher dimensions and $\$ 6$ contains a discussion of the two methods. Once we know that 0 cannot be a critical value, it is easy to perturb to obtain (E1) and (E2) and this is done in \$7.

Finally we list here some standard results about Sobolev spaces and pseudodifferential operators which will be needed in the proofs. More details can be found in [3], [6] and [8].

Norms. Recall that the topology on $C^{\infty}(M)$ is given by the countable family of $C^{k}$ norms on $M$, given as follows. For $U \subset R^{v}$ and $u \in C^{\infty}(U)$ with compact support,

$$
|u|_{k, U}=\sup _{x \in U} \sum_{|\alpha|<k}\left|D^{\alpha} u(x)\right|
$$

gives the $C^{k}$-norm on $U$ and this is globalized to the norm $|\ldots|_{k, M}$ or simply $|\ldots|_{k}$ on $M$ by a partition of unity. Similarly,

$$
\|u\|_{s, U}=\sum_{|\alpha|<s}\left(\int_{U}\left|D^{\alpha} u\right|^{2}\right)^{1 / 2}
$$

gives the $H^{s}$ norm on $U$ and this is globalized to the norm $\|\ldots\|_{s, M}$ or just $\|\ldots\|_{s}$ on $M .\|\ldots\|=\|\ldots\|_{0}$ is the usual $L^{2}$ norm on $M$.

Pseudo differential inverse. Let $P$ be a second order, selfadjoint, $C^{\infty}$ linear elliptic partial differential operator on $M$. Let $N=\operatorname{ker} P \subset C^{\infty}(M) ; \pi_{N}$ denotes projection on $N$ in $L_{2}(M)$. Then there is a pseudodifferential operator $Q$ of order -2 such that $Q P=I-\pi_{N}$ on $C^{\infty}(M)$ and $Q \pi_{N}=0$ on $L_{2}(M)$. Furthermore, $Q u(x)=\int_{M} K(x, y) u(y) d y$, where the kernel $y \rightarrow K(x, y)$ belongs to $L_{p}(M)$ for fixed $x \in M$, with $p=2$ if $\nu=2, p=(\nu-1) /(\nu-2)$ if $\nu>2$.

Facts. (1) (Sobolev's lemma) If $t>s+\nu / 2,|u|_{s} \leqslant$ (const) $\|u\|_{t}$ for $u \in$ $C^{\infty}(M)$.

(2) $\|u v\|_{s} \leqslant$ (const) $|u|_{s}\|v\|_{s}$ for $u, v \in C^{\infty}(M)$.

(3) If $Q$ is a pseudodifferential operator of order $m,\|Q u\|_{s} \leqslant$ (const) $\|u\|_{s+m}$.

2. The object of this section is to prove Theorem 1.1, which essentially says that properties E1, E2 and E3 are stable under small perturbations of the operator. It is well known from the Morse theory that E1 and E2 are stable 
properties of functions and it is easy to show that E3 is stable. The remainder of the proof consists of showing that the eigenfunctions of $L+\rho$ depend continuously on $\rho$ (Theorem 2.1 ).

We will always deal with one eigenvalue $\lambda$ of $L+\rho$ at a time and eigenvalues $\leqslant \lambda$ will be assumed to be simple. If $\lambda=\lambda_{n}$ is the $n$th eigenvalue of $L+\rho$, by the corresponding eigenvalue $\lambda^{\prime}$ of a perturbed operator $L+\rho^{\prime}$ we mean the $n$th eigenvalue $\lambda_{n}^{\prime}$. By a normalized eigenfunction $u$, we mean $\|u\|=1$.

THEOREM 2.1. Let $\rho \in B_{n}$ and let $u$ be a normalized eigenfunction of $L+\rho$ with eigenvalue $\lambda$. Let $s, t$ be nonnegative integers with $s>t+\nu / 2$. For each $\varepsilon>0$ there is a $\delta>0$ such that if $\left|\rho^{\prime}-\rho\right|_{s}<\delta$ then $\left|u^{\prime}-u\right|_{t}<\varepsilon$ for one of the (two) normalized eigenfunctions of $L+\rho^{\prime}$ whose eigenvalue $\lambda^{\prime}$ corresponds to $\lambda$.

Proof. Let $\rho^{\prime} \in C^{\infty}(M)$ and let $u^{\prime}$ be either normalized eigenfunction of $L+\rho^{\prime}$ with eigenvalue $\lambda^{\prime}$. From $(L+\rho) u=\lambda u,\left(L+\rho^{\prime}\right) u^{\prime}=\lambda^{\prime} u^{\prime}$, we get

$$
(L+\rho-\lambda)\left(u^{\prime}-u\right)=r u^{\prime}
$$

where $r=\rho-\rho^{\prime}+\lambda^{\prime}-\lambda$. Note that $|r|_{s} \leqslant 2\left|\rho^{\prime}-\rho\right|_{s}$ (see [1]). Since $\lambda$ is simple,

$$
u^{\prime}-u=Q_{\lambda}\left(r u^{\prime}\right)+\eta u
$$

for some $\eta \in \mathbf{R}$, where $Q_{\lambda}$ is the pseudo differential inverse $L+\rho-\lambda$.

Rewriting (1) as

$$
u^{\prime}=Q_{\lambda}\left(r u^{\prime}\right)+(1+\eta) u
$$

we have a decomposition of $u^{\prime}$ into components in and orthogonal to $\operatorname{ker}(L+\rho-\lambda)$. By the Pythagorean theorem in $L_{2}$,

$$
1=\left\|Q_{\lambda}\left(r u^{\prime}\right)\right\|^{2}+(1+\eta)^{2}
$$

or

$$
\eta^{2}+2 \eta+\left\|Q_{\lambda}\left(r u^{\prime}\right)\right\|^{2}=0
$$

If $\left\|Q_{\lambda}\left(r u^{\prime}\right)\right\|<1$, this has roots $\eta_{1}=-1+\sqrt{1-\left\|Q_{\lambda}\left(r u^{\prime}\right)\right\|^{2}}$ and $\eta_{2}=-1-\sqrt{1-\left\|Q_{\lambda}\left(r u^{\prime}\right)\right\|^{2}}$ which satisfy $0 \geqslant \eta_{1}>-1>\eta_{2} \geqslant-2$. But

$$
\begin{aligned}
\left\|Q_{\lambda}\left(r u^{\prime}\right)\right\| & \leqslant c_{1}\left\|r u^{\prime}\right\| \leqslant c_{1}|r|_{0}\left\|u^{\prime}\right\| \\
& \leqslant c_{1}|r|_{s} \text { since }\left\|u^{\prime}\right\|=1 \\
& \leqslant 2 c_{1}\left|\rho^{\prime}-\rho\right|_{s}<2 c_{1} \delta
\end{aligned}
$$

so $\left\|Q_{\lambda}\left(r u^{\prime}\right)\right\|<1$ provided $\delta<1 / 2 c_{1}$, which we assume from now on. Also if $\eta=\eta_{2}$ in (2), then $\eta=\eta_{1}$ in the corresponding decomposition for $-u^{\prime}$. This is obtained by multiplying (2) by -1 and using $\eta_{1}+\eta_{2}=-2$ from (3). The 
eigenfunction sought in the theorem will be the one corresponding to $\eta=\eta_{1}$, which will be our $u^{\prime}$ from now on. Finally note that

$$
\left|\eta_{1}\right|<\left|\eta_{1} \eta_{2}\right|=\left\|Q_{\lambda}\left(r u^{\prime}\right)\right\|<2 c_{1} \delta<1 .
$$

Next we start the estimate for $\left|u^{\prime}-u\right|_{t}$ using the right side of (1). $c_{1}, c_{2}$, etc., will be positive constants depending on $\rho, \lambda, u$ and $s$.

$$
\begin{aligned}
\left|u^{\prime}-u\right|_{t} & \leqslant c_{2}\left\|u^{\prime}-u\right\|_{s} \quad \text { by Sobolev's lemma, since } s>t+\nu / 2 \\
& \leqslant c_{2}\left(\left\|Q_{\lambda}\left(r u^{\prime}\right)\right\|_{s}+\left|\eta_{1}\right|\|u\|_{s}\right) \quad \text { by (1) } \\
& \leqslant c_{2}\left(1+\|u\|_{s}\right)\left\|Q_{\lambda}\left(r u^{\prime}\right)\right\|_{s} \quad \text { by (4) } \\
& \leqslant c_{3}|r|_{s}\left\|u^{\prime}\right\|_{s} \leqslant 2 c_{3} \delta\left\|u^{\prime}\right\|_{s} .
\end{aligned}
$$

LeMma 2.2. If $\delta<1 / 4 c_{3}$, then $\left\|u^{\prime}\right\|_{s} \leqslant c_{5}$.

$$
\begin{aligned}
\left\|u^{\prime}\right\|_{s} & \leqslant\left\|u^{\prime}-u\right\|_{s}+\|u\|_{s} \\
& \leqslant 2 c_{4} \delta\left\|u^{\prime}\right\|+\|u\|_{s} \text { from above } \\
& \leqslant \frac{1}{2}\left\|u^{\prime}\right\|_{s}+\|u\|_{s} \text { if } \delta<1 / 4 c_{4} ;
\end{aligned}
$$

hence

$$
\left\|u^{\prime}\right\|_{s} \leqslant 2\|u\|_{s}=c_{5} .
$$

Returning to (5), $\left|u^{\prime}-u\right|_{t} \leqslant 2 c_{3} c_{5} \delta<\varepsilon$, provided $\delta<\min \left(\varepsilon / 2 c_{3} c_{5}, 1 / 4 c_{4}\right.$, $\left.1 / 2 c_{1}\right)$, so the theorem is proved.

Proposition 2.3. If $U \subset M$ is an open set containing all the nodal critical points of $u \in C^{\infty}(M)$, then $\exists \varepsilon>0$ such that if $v \in C^{\infty}(M),|v-u|_{1}<\varepsilon$, then all the nodal critical points of $v$ lie in $U$ also.

CoROLlary. If $u$ has no nodal critical points, $\exists \varepsilon>0$ such that $|v-u|_{1}<\varepsilon$ implies $v$ has no nodal critical points. (Apply the proposition with $U=\varnothing$.)

Proof of the Proposition. For $u \in C^{\infty}(M), p \in M$, define $u_{(1)}(p)=$ $|u(p)|+|d u(p)|$ where the norm $|d u(p)|$ comes from a (fixed) metric on the cotangent bundle of $M$. Then (i) $u_{(1)}(p)=0$ if and only if $p$ is a nodal critical point of $u$; (ii) $(u+v)_{(1)}(p) \leqslant u_{(1)}(p)+v_{(1)}(p)$; (iii) $|u|_{(1)}=\sup _{p \in M} u_{(1)}(p)$ is a norm on $C^{\infty}(M)$ and $|\ldots|_{(1)}$ is equivalent to $|\ldots|_{1}$, the $C^{1}$ norm. By (iii) it suffices to prove the proposition using $|\ldots|_{(1)}$. Let $V_{\delta}=\left\{p \in M: u_{(1)}(p)\right.$ $<\delta\}$.

Claim. (1) If $|v-u|_{(1)}<\delta$, then all nodal critical points of $v$ lie in $V_{\delta}$; (2) $\exists \delta>0$ such that $V_{\delta} \subset U$. (1) and (2) prove the proposition.

PROOF OF (1). If $p$ is a nodal critical point of $v$, then $v_{(1)}(p)=0$ by (i). Then $u_{(1)}(p) \leqslant(u-v)_{(1)}(p)+v_{(1)}(p)<\delta$, hence $p \in V_{\delta}$.

Proof of (2). If $U=M, \delta$ is arbitrary. For $U \neq M$ let $\delta=\inf \left\{u_{(1)}(p)\right.$ : 
$p \notin U\}$. For each $p \notin U, u_{(1)}(p)>0$ since all the nodal critical points of $u$ lie in $U$. But $u_{(1)}$ is a continuous function, hence it attains its minimum on the nonempty compact set $M-U$; thus $\delta>0$. Finally $p \notin U$ implies $u_{(1)}(p)>$ $\delta$, so $p \notin V_{\delta}$. Thus $V_{\delta} \subset U$.

The following lemma is standard Morse theory.

LEMMA 2.5. $\left\{u \in C^{\infty}(M): u\right.$ is a Morse function and distinct critical points of $u$ have distinct critical values $\}$ is open and dense in $C^{\infty}(M)$.

Proof OF TheORem 1.1. Use induction on $n$. The theorem is true for $n=0$ since $B_{0}=C^{\infty}(M)$. Assume $n \geqslant 1$ and let $\rho \in B_{n}$. We will show $\exists \delta>0$ and a nonnegative integer $s$ such that $\left|\rho^{\prime}-\rho\right|_{s}<\delta$ implies $\rho^{\prime} \in B_{n}$. Since $\rho \in B_{n}$ $\subset A_{n} \cap B_{n-1}$ which is open, $\exists \delta_{1}>0$ and $s_{0}>0$ such that $\left|\rho^{\prime}-\rho\right|_{s_{0}}<\delta_{1}$ implies $\rho^{\prime} \in A_{n} \cap B_{n-1}$. Also since $s>s_{0} \Rightarrow\left|\rho^{\prime}-\rho\right|_{s_{0}}<\left|\rho^{\prime}-\rho\right|_{s}$ we may replace $s_{0}$ by a larger $s$ when necessary. To show $\rho^{\prime} \in B_{n}$ it is now only necessary to check the $n$th eigenspace. Let $u_{n} \in \operatorname{ker}\left(L+\rho-\lambda_{n}\right),\left\|u_{n}\right\|=1 . u_{n}$ satisfies E1, E2 and E3 since $\rho \in B_{n}$. By the corollary and lemma above, $\exists \varepsilon>0$ and a positive integer $t$ such that $\left|v-u_{n}\right|_{t}<\varepsilon$ implies $v$ satisfies E1, E2, E3. By Theorem 2.1, for any $s>t+\nu / 2, \exists \delta_{2}>0$ such that $\left|\rho^{\prime}-\rho\right|_{s}<$ $\delta_{2}$ implies $\exists u_{n}^{\prime}$ with $\left|u_{n}^{\prime}-u_{n}\right|_{t}<\varepsilon$; hence the functions in $\operatorname{ker}\left(L+\rho^{\prime}-\lambda_{n}^{\prime}\right)$ satisfy E1, E2, and E3. Take $\delta=\min \left(\delta_{1}, \delta_{2}\right)$ and the theorem is proved.

3. The analytic proof that one can perturb away the nodal critical points (\$4) requires some detailed information about the structure of the zero set near a nodal critical point. The restriction to 2 dimensions enters here because of the use of complex variables in the proof of Lemma 3.4.

Definition 3.1. (1) $a \in M$ is a zero of order $k$ of $u \in C^{\infty}(M)$ if $D^{\alpha} u(a)=$ 0 for all $a$ with $|\alpha|<k$ but there is an $\alpha$ with $|\alpha|=k$ such that $D^{\alpha} u(a) \neq 0$. (2) A zero $a$ of order $k$ is weakly degenerate if there exists $c>0$ such that $|d u(x)|>c|x-a|^{k-1}$ for $x$ in a neighborhood of $a$.

In particular, if $a$ is a weakly degenerate nodal critical point (i:e., $k>2$ ) then there are no other critical points in a neighborhood of $a$.

Proposition 3.2. Let $P$ be a second order selfadjoint $C^{\infty}$ elliptic differential operator on $M$, with $\operatorname{dim} M=2$. If $P u=0$, all the zeros of $u$ are weakly degenerate.

Since $M$ is compact and nodal critical points which are weakly degenerate are isolated, $u$ has a finite number of nodal critical points. The proposition follows from the following two lemmas and the fact that every zero of $u$ has order $k$, for some $k<\infty$, by the strong unique continuation theorem [4].

Facts. (1) If $P$ is a differential operator of order $m$ and $u$ has a zero of order $k$ at $a$, then $P u$ has a zero of order $\geqslant k-m$ at $a$. 
(2) If $f \in C^{\infty}(M)$ has a zero of order $j$ and $u$ has a zero of order $k$ at $a$, then $f u$ has a zero of order $k+j$ at $a$.

(3) A nonzero polynomial in $x-a$, homogeneous of degree $k$, has a zero of order $k$ at $a$.

Lemma 3.3. Let $P$ be a second order $C^{\infty}$ elliptic differential operator on $M$ ( $\operatorname{dim} M=\nu$, arbitrary) and suppose $P u=0$. If $a \in M$ is a zero of order $k$ of $u$, there are coordinates $\left(x_{1}, \ldots, x_{\nu}\right)$ on a neighborhood $U$ of a such that the kth Taylor polynomial $u_{k}(x)=\sum_{|\alpha|=k} D^{\alpha} u(a)(x-a)^{\alpha}$ satisfies $\Delta u_{k}=0$, where $\Delta=\sum_{i=1}^{\nu} \partial^{2} / \partial x_{i}^{2}$ is the Laplacian.

Proof. If $k=0$ or 1 , this is true for any coordinates, for $\Delta$ annihilates linear functions. Assume $k \geqslant 2$. One can choose coordinates $\left(x_{1}, \ldots, x_{\nu}\right)$ on a neighborhood $U$ of $a$ so that the principal part of $P$ is $\Delta$, i.e. if

$$
P=\sum_{i, j=1}^{\nu} g_{i j} \partial^{2} / \partial x_{i} \partial x_{j}+T
$$

where $T$ is a first order operator, then $g_{i j}(a)=\delta_{i j}$. Thus

$$
P=\Delta+\sum_{i j=1}^{\nu}\left[g_{i j}-\delta_{i j}\right] \partial^{2} / \partial x_{i} \partial x_{j}+T .
$$

Let $v=u-u_{k} ; v$ has a zero of order $\geqslant k+1$ at $a$. We have

$$
\begin{aligned}
0 & =P u=\Delta u_{k}+\Delta v+\sum_{i, j=1}^{\nu}\left[g_{i j}-\delta_{i j}\right] \partial^{2} u / \partial x_{i} \partial x_{j}+T u \\
& =\Delta u_{k}+f .
\end{aligned}
$$

Since $u_{k}$ is a homogeneous polynomial of degree $k, \Delta u_{k}$ is a homogeneous polynomial of degree $k-2$, hence either $\Delta u_{k}=0$ or $\Delta u_{k}$ has a zero of order $k-2$ at $a$. But $f$ has a zero of order $\geqslant k-1$, which implies $\Delta u_{k}=0$. To see this, note: (1) $v$ has a zero of order $\geqslant k+1$ and $\Delta$ is of order 2 , so $\Delta v$ has a zero of order $\geqslant k-1$; (2) $u$ has a zero of order $k$ and $T$ has order 1 so $T u$ has a zero of order $\geqslant k-1$; (3) $\partial^{2} u / \partial x_{i} \partial x_{j}$ has a zero of order $k-2$, but $g_{i j}-\delta_{i j}$ vanishes at $a$ (zero of order $\geqslant 1$ ), so $\sum_{i j=1}^{\nu}\left[g_{i j}-\delta_{i j}\right] \partial^{2} u / \partial x_{i} \partial x_{j}$ has a zero of order $\geqslant k-1$.

This lemma is not new; see Bers [5] for a similar result with weaker smoothness assumptions on the coefficients of the operator.

LEMMA 3.4. Assume $\operatorname{dim} M=2$. Suppose that $u$ has a zero of order $k$ at a and there are coordinates about $a$ in which $\Delta u_{k}=0$. Then $a$ is a weakly degenerate zero of $u$.

Proof. Since $\operatorname{dim} M=2$, we can use complex variables. Let $z=x_{1}+x_{2} \sqrt{-1}$. Since $\Delta u_{k}=0, u_{k}=\operatorname{Re} f$ for some complex analytic 
function $f$. Requiring $f(a)=0$, which we may do, implies that $f$ is homogeneous of degree $k$ in $z-a$. Hence $f(z)=\xi(z-a)^{k}$ for some complex $\xi$, so

$$
u_{k}(z)=\frac{1}{2}\left[\xi(z-a)^{k}+\bar{\xi}(\bar{z}-\bar{a})^{k}\right] .
$$

Now $\left|d u_{k}(x)\right|=2\left|\partial u_{k}(z) / \partial z\right|$ where

$$
\frac{\partial}{\partial z}=\frac{1}{2}\left(\frac{\partial}{\partial x_{1}}-\sqrt{-1} \frac{\partial}{\partial x_{2}}\right)
$$

But $\partial u_{k} / \partial z=k \xi(z-a)^{k-1} / 2$, so

$$
\left|d u_{k}(x)\right|=k|\xi||z-a|^{k-1} \geqslant k|\xi||x-a|^{k-1} .
$$

Finally $u=u_{k}+v$, so

$$
\begin{aligned}
|d u(x)| & \geqslant\left|d u_{k}(x)\right|-|d v(x)| \\
& \geqslant k|\xi||x-a|^{k-1}-c|x-a|^{k} \text { since } v \text { has a zero of order } k+1 \\
& \geqslant \frac{1}{2}|\xi||x-a|^{k-1}
\end{aligned}
$$

provided $|x-a| \leqslant k|\xi| / 2 c$.

4. This section contains essentially the original proof (the first proposition is strengthened slightly for use in $\$ 5$ ) that the nodal critical points can be perturbed away to obtain (E3). The proof uses the results of $\$ 3$ heavily, so is only valid for $\operatorname{dim} M=2$; see $\$ 6$ for comments. Let $\rho \in A_{n} \cap B_{n-1}$ and let $\lambda$ be the $n$th eigenvalue of $L+\rho, u$ an eigenfunction with eigenvalue $\lambda$. Consider a linear perturbation $\rho(\tau)=\rho-\tau \sigma$ where $\tau \in \mathbf{R}$ and $\sigma \in C^{\infty}(M)$. As in [1], [3], we get $\lambda(\tau)=\lambda+\tau \alpha+\tau^{2} \beta(\tau)$ and $u(\tau)=u+\tau v+\tau^{2} w(\tau)$ using Rellich's theorem [7]. Equating the coefficient of $\tau$ to zero in the perturbation equation $(L+\rho+\tau \sigma) u(\tau)=0$ gives $(L+\rho-\lambda) v=(\sigma+\alpha) u$. Using the pseudo-inverse $Q_{\lambda}$ (see $\S 1$ ) gives

$$
v=Q_{\lambda}(\sigma u)+\eta u \text { for some } \eta \in \mathbf{R}
$$

i.e.

$$
v(x)=\int K(x, y) \sigma(y) u(y) d y+\eta u(x) .
$$

Proposition 4.1. There exists $\sigma \in C^{\infty}(M)$ such that $v(x) \neq 0$ for all $x$ with $u(x)=0$.

In the proof of the proposition, we use the fact that the zero set $u^{-1}(0)$ has measure zero. This is another consequence of the unique continuation theorem [4]. For if

$$
G_{\alpha j}=\left\{x: D^{\alpha} u(x)=0 \text { but }\left(\partial D^{\alpha} u / \partial x_{j}\right)(x) \neq 0\right\}
$$

then $G_{\alpha_{j}}$ is a submanifold of dimension $\nu-1$ so has measure zero and 
$u^{-1}(0)=\cup_{\alpha} \cup_{j=1}^{v} G_{\alpha j}$ is a countable union, so it has measure zero.

Proof of PROPOSITION. Choose $w \in N^{\perp} \cap C^{\infty}(M)$ with $w(x) \neq 0$ for all $x \in u^{-1}(0)$. Such a $w$ exists since if $g \in C^{\infty}(M)$ satisfies $g(x)=0$ for $x \in u^{-1}(0)$, then $\pi_{N^{\perp}} g=g-\theta u \in N^{\perp} \cap C^{\infty}(M)$ satisfies the same property. Since $Q_{\lambda}$ is onto $N^{\perp}$, there is a function $f \in C^{\infty}(M)$ such that $w(x)=Q_{\lambda}(f)=\int_{M} K(x, y) f(y) d y$. Let $J=\inf \left\{|w(x)|: x \in u^{-1}(0)\right\}$. Then $J>0$ since $u^{-1}(0)$ is closed, hence compact. Recall $K_{x}: y \rightarrow K(x, y)$ is in $L_{p}$ for an appropriate $p$ given above. Since $u^{-1}(0)$ has measure zero, we can choose an open neighborhood $N$ of $u^{-1}(0)$ such that $\mu(N) \leqslant$ $\left(J /\left(2\left\|K_{x}\right\|_{p}|f|_{0}\right)\right)^{p}$, where $\mu$ is the measure (Lebesgue) on $M$ and $q$ is conjugate to $p$. Let $\phi \in C^{\infty}(M)$ be a bump function satisfying $0 \leqslant \phi \leqslant 1$, and $\phi=0$ on a neighborhood of $u^{-1}(0)$ whose closure is contained in $N$ and $\phi=1$ outside $N$. Then

$$
\sigma= \begin{cases}\phi f / u & \text { outside } u^{-1}(0), \\ 0 & \text { on } u^{-1}(0),\end{cases}
$$

is a well-defined $C^{\infty}$ function. Let $v(x)=\int_{M} K(x, y) \sigma(y) u(y) d y$. Then

$$
\begin{aligned}
w(x)-v(x) & =\int_{M} K(x, y)[f(y)-\sigma(y) u(y)] d y \\
& =\int_{N} K(x, y) f(y)[1-\phi(y)] d y
\end{aligned}
$$

and

$$
\begin{aligned}
|w(x)-v(x)| & \leqslant\left[\int_{N}|K(x, y)|^{p} d y\right]^{1 / p}\left[\int_{N}|f(y)[1-\phi(y)]|^{q} d y\right]^{1 / q} \\
& \leqslant\left\|K_{x}\right\|_{p} \mid f_{0} \mu(N)^{1 / q} \quad \text { since } 0 \leqslant 1-\phi \leqslant 1 \\
& \leqslant J / 2 \text { by the choice of } N \text { above. }
\end{aligned}
$$

Thus for $x \in u^{-1}(0)$,

$$
\begin{aligned}
|v(x)| & \geqslant|w(x)|-|w(x)-v(x)| \\
& \geqslant J-J / 2=J / 2>0 .
\end{aligned}
$$

ReMARK. Essentially what we have shown is that $\left\{Q_{\lambda}[\sigma u]: \sigma \in C^{\infty}(M)\right\}$ is dense in $N^{\perp} \cap C^{\infty}(M)$ with respect to the $C^{0}$ norm.

Proposition 4.2. For $\tau \neq 0$ in a small neighborhood of zero, $u(\tau)$ has no nodal critical points.

Proof. By the results in $\$ 3$, the original eigenfunction $u$ has a finite number of nodal critical points $a_{1}, \ldots, a_{n}$. (The argument for $n=1$ is given in [2].) By the preceding proposition, we can choose $\sigma$ so that the corresponding $v$ satisfies $v\left(a_{i}\right) \neq 0$ for $i=1, \ldots, n$. Choose neighborhoods $V_{i}$ of $a_{i}$ so 
that (1) $|v(x)| \geqslant J>0$ for all $x \in V=\cup_{i=1}^{n} V_{i}$ and (2) on $V_{i}$, we have $|u(x)| \leqslant c_{i}|x-a|^{k_{i}},|d u(x)| \geqslant c_{i}^{\prime}|x-a|^{k_{i}-1}$. (2) says that the nodal critical point $a_{i}$ is a weakly degenerate zero of order $k_{i} ; \S 3$ justifies this assumption. By continuity, for any sufficiently small neighborhood of $\tau=0$ we have (3) $|v(\tau)(x)| \geqslant J / 2$ for $x$ in $V$, where $v(\tau)=v+\tau w(\tau)$, and (4) $u(\tau)$ has no nodal critical points outside $V$. But $u(\tau)$ has no nodal critical points inside $V$ either, for if $x \in V_{i}$ is a critical point of $u(\tau)$, we have

$$
0=[d u(\tau)](x)=d u(x)+\tau[d v(\tau)](x)
$$

and by (2) above

$$
\left|x-a_{i}\right|^{k_{i}-1} \leqslant|d u(x)| / c_{i}^{\prime}=|\tau||d v(\tau)(x)| / c^{\prime}{ }_{i} \leqslant c_{i}^{\prime \prime}|\tau|
$$

and

$$
\begin{aligned}
|u(\tau)(x)| & =|u(x)+\tau v(\tau)(x)| \\
& \geqslant|\tau||v(\tau)(x)|-|u(x)| \\
& \geqslant J|\tau| / 2-h|x-a|^{k_{i}} \\
& \geqslant J|\tau| / 2-h\left(c_{i}^{\prime \prime}\right)^{1 /\left(k_{i}-1\right)}|\tau|^{k_{i} /\left(k_{i}-1\right)} \\
& \geqslant J|\tau| / 4 \text { provided }|\tau| \leqslant(J / 4 h)^{k_{i}-1} / c_{i}^{\prime \prime} \\
& \neq 0 \quad \text { if }|\tau| \neq 0 .
\end{aligned}
$$

Thus if $\tau$ is small enough and nonzero there are no nodal critical points of $u(\tau)$.

5. One can also use Sard's theorem to prove that the nodal critical points can be perturbed away. This proof works in all dimensions. We give a direct proof here, which is essentially a very simple special case of one of $\mathrm{K}$. Uhlenbeck's iransversality results [9], [10]. The idea for completing the higher dimensional case in this way came from reading her paper and trying to see just where the two approaches differed (see $\S 6$ ).

Using Proposition 4.1, let $\sigma$ be such that $v(x) \neq 0$ on $u^{-1}(0)$.

Proposition 5.1. For such a $\sigma$, the set of $\tau$ 's close to zero with $u(\tau)$ having no nodal critical points is the complement of a set of measure zero.

Proof. Let $\phi: \mathbf{R} \times M \rightarrow \mathbf{R}$ be given by $\phi(\tau, x)=u(\tau)(x)$. The equation $\phi(\tau, x)=0$ gives the zeros of $u(\tau)$. Using the implicit function theorem, this equation can be solved for $\tau$ as a function of $x$ near $\tau=0$. For $\phi(0, x)=0$ corresponds to $u(x)=0$, so $\partial \phi(0, x) / \partial \tau=v(x) \neq 0$ by the choice of $\sigma$. Thus there is a neighborhood $N_{x}$ of each $x \in u^{-1}(0)$ and a $C^{\infty}$ function $f: N_{x} \rightarrow \mathbf{R}$ such that near $(0, x), \phi^{-1}(0)=\left\{\left(f_{x}(y), y\right): y \in N_{x}\right\}$. By compactness of $M$, there are neighborhoods $V$ of $\tau=0$ and $N$ of $u^{-1}(0)$ and a function $f: N \rightarrow \mathbf{R}$ 
such that $\phi^{-1}(0) \cap V=\{(f(x), x): x \in N\}$. For $x \in N, \phi(f(x), x)=0$, and differentiating with respect to $x$ yields $(\partial \phi / \partial \tau) \cdot d f+d[u(\tau)]=0$. But $x$ is a nodal critical point of $u(\tau)$ if $\phi(\tau, x)=0$ and $d[u(\tau)](x)=0$. Since $\partial \phi / \partial \tau \neq$ 0 on $V, x$ is a nodal critical point if and only if $d f(x)=0$, i.e. $x$ is a critical point of $f$. This implies $u(\tau)$ has nodal critical points if and only if $\tau$ is a critical value of $f$. But by Sard's theorem, the set of critical values of a $C^{\infty}$ function has measure zero.

6. Some comments on the two preceding sections are in order. In the proof of $\$ 4$, we used heavily the fact that solutions of second order elliptic equations in 2 dimensions have only weakly degenerate zeros. It would be nice if this were true in higher dimentions but it is not. For example, view the circle $S^{1}$ as $[0,2 \pi]$ with endpoints identified and take

$$
u\left(x_{1}, x_{2}, x_{3}\right)=\sin x_{1} \sin x_{2} \sin x_{3}
$$

as an eigenfunction of

$$
\Delta=\frac{\partial^{2}}{\partial x_{1}^{2}}+\frac{\partial^{2}}{\partial x_{2}^{2}}+\frac{\partial^{2}}{\partial x_{3}^{2}}
$$

on $S^{1} \times S^{1} \times S^{1} .(0,0,0)$ is a nodal critical point which is not isolated, hence not weakly degenerate. Thus it is not possible to extend this proof to higher dimensions without more information on the growth of eigenfunctions near nodal critical points.

The reason why this method works only in such a special case, in contrast to the method of $\$ 5$ and transversality methods in general, may be related to the fact that it actually proves slightly more. What was shown in $\$ 4$ (for $\operatorname{dim} M=2$ ) was that passing through each $\rho \in C^{\infty}(M)$ there is a family of line segments $\rho^{\prime}=\rho+\tau \sigma$, forming a "star" as in the diagram, such that for any $\rho^{\prime} \neq \rho$ in this set, the $n$th eigenfunction has no nodal critical points. The result in $\$ 5$ shows that along each segment in the star, only certain $\tau$ 's (the complement of a set of measure zero) work.

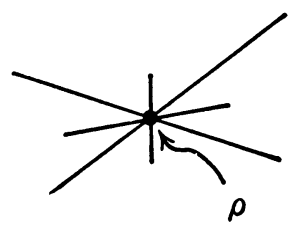

This comparison is interesting also from a constructive viewpoint. Again assuming we can find $\sigma$, in two dimensions we can explicitly write down $\rho^{\prime}$ because of $\$ 4$ whereas in higher dimensions we cannot in general. There is an extremely important special case for which we do have the same "star" of admissible perturbations in all dimensions. If the manifold and the operator 
are analytic, so are the eigenfunctions $u(\tau)$. Since $u(\tau)$ is analytic in $\tau$, $\phi(\tau, x)=u(\tau)(x)$ is analytic in $(\tau, x)$. The implicit function theorem then implies $\tau=f(x)$ (see \$5) is an analytic function of $x$. But the critical values of a real-analytic function on a compact manifold are isolated [3]. Hence there is an interval about $\tau=0$ for which $u(\tau)$ has no nodal critical points.

7. Once the nodal critical points are eliminated, the perturbation yielding (E1) and (E2), i.e. nondegenerate critical points with distinct critical values, is fairly straightforward. Suppose $(L+\rho=\lambda u$ with $\lambda$ simple and $u$ having no nodal critical points. We want to prou e e $\rho^{\prime}$ near $\rho$ whose corresponding eigenfunction satisfies (E2) and (E3). To do this, we construct $u^{\prime}$ first and show that it is an eigenfunction of an appropriate $\rho^{\prime}$ (with the same eigenvalue $\lambda$ ).

First choose a bump function $\phi \in C^{\infty}(M), 0 \leqslant \phi \leqslant 1$, with $\phi=1$ on a neighborhood of the nodal set $u^{-1}(0)$ and $\phi=0$ on a neighborhood of the critical point set $d u^{-1}(0)$ of $u$. Let

$$
\gamma_{1}=\inf \left\{|u(x)|: x \notin \phi^{-1}(1)\right\}, \quad \gamma_{2}=\inf \left\{|d u(x)|: x \notin \phi^{-1}(0)\right\}
$$

and

$$
\gamma=\min \left\{\gamma_{1}, \gamma_{2}\right\}
$$

Then $\gamma>0$; e.g., since $\phi=1$ on a neighborhood of $u^{-1}(0), \gamma_{1}>0$ and similarly for $\gamma_{2}$.

LEMMA 7.1. Given a function $v$ such that $|v-u|_{1}<\gamma / 2$, if $u^{\prime}=\phi u+(1-$ $\phi) v$, then the zero sets of $u^{\prime}$ and $u$ coincide and the critical point sets of $u^{\prime}$ and $v$ coincide.

Proof. We show the statement about the zero sets. First, $u^{\prime}$ has no zeros when $\phi \neq 1$, for then $|u| \geqslant \gamma_{1} \geqslant \gamma$ and

$$
\left|u-u^{\prime}\right|=|(1-\phi)(v-u)| \leqslant|v-u|<\gamma / 2,
$$

so

$$
\left|u^{\prime}\right| \geqslant|u|-\left|u-u^{\prime}\right| \geqslant \gamma-\gamma / 2=\gamma / 2>0 .
$$

Second, when $\phi=1, u^{\prime}=u$ so the only zeros of $u^{\prime}$ occur at the zeros of $u$. The other statement is proved similarly, with $u^{\prime}$ replaced by $d u^{\prime}$, etc.

One consequence of the lemma is that if $v$ satisfies (E2) and (E3), then $u^{\prime}$ satisfies (E1), (E2) and (E3) since $u$ has no critical points in the neighborhood $\{\phi=1\}$ of its zero set and $u^{\prime}=u$ there. By the Morse lemma we can find such a $v$ as close as we want to $u$. A second consequence is that we can define $\rho^{\prime}$ as follows. Let

$$
\rho^{\prime}= \begin{cases}\left(\lambda u^{\prime}-L u^{\prime}\right) / u^{\prime} & \text { when } u \neq 0 \\ \rho & \text { when } \phi=1\end{cases}
$$


Since $u^{\prime} \neq 0$ whenever $u=0$ by the lemma, the first part is well defined. Also, the two parts agree on the overlap $\{x: \phi(x)=1$ and $u(x) \neq 0\}$ since $u^{\prime}=u \neq 0$ there and $(\lambda u-L u) / u=\rho$. So $\rho^{\prime}$ is well defined and $C^{\infty}$. Furthermore, $u^{\prime}$ is clearly an eigenfunction of $L+\rho^{\prime}$ with eigenvalue $\lambda$. It remains to show that by choosing $v$ close to $u$, we get $\rho^{\prime}$ close enough to $\rho$. Let $V=\{x: u(x) \neq 0\}$. Then

$$
\rho^{\prime}-\rho=\frac{\lambda u^{\prime}-L u^{\prime}}{u^{\prime}}-\rho=\frac{(L+\rho-\lambda)\left(u-u^{\prime}\right)}{u^{\prime}}
$$

since $(L+\rho-\lambda) u=0$. Thus

$$
\begin{aligned}
\left|\rho^{\prime}-\rho\right|_{s, V} & \leqslant c_{1}\left|\frac{1}{u^{\prime}}\right|_{s, V}\left|(L+\rho-\lambda)\left(u-u^{\prime}\right)\right|_{s, V} \\
& \leqslant c_{2}\left|\frac{1}{u^{\prime}}\right|_{s, V}\left|u-u^{\prime}\right|_{s+2, V} \\
& \leqslant c_{3}\left|\frac{1}{u^{\prime}}\right|_{s, V}|u-v|_{s+2, V}
\end{aligned}
$$

Now

$$
\begin{aligned}
\left|\frac{1}{u^{\prime}}\right|_{s, V} & \leqslant\left|\frac{1}{u}\right|_{s, V}+\left|\frac{u-u^{\prime}}{u u^{\prime}}\right|_{s, V} \\
& \leqslant\left|\frac{1}{u}\right|_{s, V}+c_{4}\left|\frac{1}{u}\right|_{s, V}\left|\frac{1}{u^{\prime}}\right|_{s, V}\left|u-u^{\prime}\right|_{s, V} \\
& \leqslant\left|\frac{1}{u}\right|_{s, V}+c_{5}\left|\frac{1}{u^{\prime}}\right|_{s, V}|u-v|_{s, V}
\end{aligned}
$$

Thus if

$$
|u-v|_{s, V} \leqslant 1 / 2 c_{5}\left|1 / u^{\prime}\right|_{s, V} \leqslant 2|1 / u|_{s, V}
$$

and

$$
\left|\rho^{\prime}-\rho\right|_{s, V} \leqslant c_{6}|u-v|_{s+2, V}
$$

But since $\rho^{\prime}=\rho$ on a neighborhood $\phi^{-1}(1)$ of $u^{-1}(0),\left|\rho^{\prime}-\rho\right|_{s} \leqslant c_{6} \mid u-$ $\left.v\right|_{s+2}$ on all of $M$.

Thus given $\varepsilon>0$, choose $v$ satisfying E2, E3 such that $|u-v|_{s+2}<$ $\min \left\{\varepsilon / c_{6}, 1 / 2 c_{5}, \gamma / 2\right\}$ by Lemma 2.5. Then $u^{\prime}=\phi u+(1-\phi) v$ satisfies El, E2, E3 and is an eigenfunction of $L+\rho^{\prime}, \rho^{\prime}$ as given above, with eigenvalue $\lambda$ and $\left|\rho^{\prime}-\rho\right|_{s}<\varepsilon$. Hence $B_{n}$ is dense in $A_{n} \cap B_{n-1}$.

ACKNOWLEDGEMENTS. The author would like once again to thank V. Guillemin and I. M. Singer for help and guidance with the thesis from which much of this paper comes; and $\mathrm{K}$. Uhlenbeck for extensive correspondence regarding her work. 


\section{REFERENCES}

1. J. H. Albert, Genericity of simple eigenvalues for elliptic PDE's, Proc. Amer. Math. Soc. 48 (1975), 413-418.

2. Nodal and critical sets for eigenfunctions of elliptic operators, Proc. Sympos. Pure Math., vol. 23, Amer. Math. Soc., Providence, R. I., 1973, pp. 71-78.

3. _ Topology of the nodal and critical point sets for eigenfunctions of elliptic operators, Thesis, M.I.T., Cambridge, Mass., 1971.

4. N. Aronszajn, $A$ unique continuation theorem for solutions of elliptic partial differential equations or inequalities of the second order, J. Math. Pures Appl. 36 (1957), 235-247.

5. L. Bers, Local behavior of solutions of general linear elliptic equations, Comm. Pure Appl. Math. 8 (1955), 473-496.

6. L. Bers, F. John and M. Schechter, Partial differential equations, Lectures in Applied Math., vol. 3, Interscience, New York, 1964.

7. F. Rellich, Perturbation theory of eigenvalue problems, Gordon and Breach, New York, 1969.

8. R. Seeley, Topics in pseudo-differential operators, Pseudo-differential operators (C.I.M.E., Stresa, 1968), Edizioni Cremonese, Rome, 1969, pp. 167-305.

9. K. Uhlenbeck, Eigenfunctions of Laplace operators, Bull. Amer. Math. Soc. 78 (1972), 1073-1076.

10.

Generic properties of eigenfunctions, Amer. J. Math. 98 (1976), 1059-1078.

Department of Mathematics, Tufts University, Medford, Massachusetts 02155 